\title{
Medizin der Zukunft: segmentiert oder vernetzt?
}

Eberhard Wolff

1 Medizin für wen? Gesundheitsversorgung im Spannungsfeld zwischen den Bedürfnissen der Patienten und der Dynamik einer marktorientierten Medizin. www.samw.ch/de/Projekte/ Oekonomisierung-der-

Medizin.html
Wie wird sich die Medizin in absehbarer Zukunft entwickeln? Das fragten vor kurzem gleich zwei Fachtagungen in Bern. «Medizin für wen?» [1] hiess es bei der Gemeinschaftsveranstaltung der Schweizerischen Akademie der Medizinischen Wissenschaften (SAMW) und der Nationalen Ethik-Kommission. Dahinter stand die Frage, welche Auswirkungen eine zunehmende Ökonomisierung der Medizin auf die Zugangsgerechtigkeit zu medizinischen Gütern habe. «Quo vadis, KVG?», fragte prosaischer die Helsana auf ihrem Zukunftssymposium aus Anlass des 15. Geburtstags des Krankenversicherungsgesetzes. So heterogen die Beiträge waren, so wenig dürfte es ein Zufall sein, dass zwei Themen auf beiden Veranstaltungen einen wichtigen Platz einnahmen: einerseits die absehbaren Herausforderungen der Medizin durch das sogenannte «Enhancement», andererseits der befürchtete Anstieg von Polymorbidität vor dem Hintergrund steigender Lebenserwartung. Und so unterschiedlich beide Themen erscheinen, haben sie eine zu beiden Anlässen diskutierte Gemeinsamkeit darin, dass sie Fragen an die Struktur des Medizinsystems stellen.

\section{Enhancement:}

\section{ein eigenständiger Medizinbereich?}

Zum sogenannten «Enhancement», der «Verbesserung» des menschlichen Körpers, zählen klassischerweise Eingriffe zur Verschönerung des Körpers. In den Schweizer Ballungszentren, nicht zuletzt am Genfersee, spriessen derzeit Botox-Praxen wie Pilze aus dem Boden. Im Zentrum von Zürich findet eine solche Praxis, nein, ein «Resort», regen Zuspruch, in dem spezialisierte Ärzte Falten weg- und Lippen mit «Filler» aufspritzen. Philippe Snozzi aus dieser Einrichtung situierte sich und seine Enhancement-Behandlungen auf der SAMW-Tagung deutlich ausserhalb einer klassischen Arztpraxis. Das Ziel sei keine Heilung von Krankheiten oder krankheitsbedingten Leiden, keine Prävention, sondern die Verbesserung des allgemeinen Lebensgefühls bzw. ein «Luxus», den sich häufig Vertreter kompetitiver Berufsgruppen, etwa Banker, Anwälte oder Ärzte, leisteten. Ihre Klientel seien keine Patienten, sondern «Kunden». Der Referent und seine Kollegen übten hier keine ärztliche Tätigkeit mehr im eigentlichen Sinn aus. Ihr Job müsse klar von der «Grundversorgung» getrennt werden. Aus diesem Grund sei die von ihnen praktizierte und von etwa drei Prozent der Schweizer Bevölkerung konsumierte «Life-
style-Medizin» weder eine Ergänzung noch eine Konkurrenz zur allgemeinen Gesundheitsversorgung.

Allerdings stiess Snozzis Modell einer klaren Aufteilung in die zwei Felder «Grundversorgung» und «Lifestyle-Medizin» bereits im Referat selbst an seine Grenzen: auch «Lifestyle-Mediziner» würden eine «ärztliche Verrichtung» ausüben, für die etwa ethische Grundregeln gelten. Der wirtschaftlich motivierten Lifestyle-Medizin liesse sich nicht einfach eine klinische Medizin ohne individuelle wirtschaftliche Überlegungen gegenüberstellen, konzedierte der Referent.

Noch deutlicher zeigten sich die fliessenden Übergänge zur Medizin im engeren Sinne am Beispiel des sogenannten «Neuroenhancements». Anne Eckhardt präsentierte auf der SAMW-Tagung eine Studie zum Gebrauch von verhaltensbeeinflussenden Medikamenten wie Ritalin. Sie dürften im Moment von ein bis zwei Prozent der Schweizer Bevölkerung regelmässig verwendet werden, häufig von Frauen im mittleren Lebensalter. Dass man sie nicht einfach als eine Art unmoralisches oder aussermedizinisches «Doping» für Aufsteiger sehen kann, zeigt der Umstand, dass im «Neuroenhancement» häufig antidepressive, angstlösende Substanzen eingenommen werden, um in der Leistungsgesellschaft mithalten zu können, speziell bei Doppelbelastung von Familie und Beruf, wie die Medizinethikerin Nikola Biller-Andorno unterstrich.

Allgemeiner formuliert: Eines der Merkmale der erblühenden «Gesundheitsgesellschaft», so Georges T. Roos auf dem Helsana-Symposium, ist das Ineinanderfliessen von kurativer Medizin, präventiver Medizin und Wellness. Auch Helsana-Verwaltungsratspräsident Thomas D. Szucs wies darauf hin, dass sich die Grenzen zwischen dem Gesundheitsmarkt und dem allgemeinen Konsum zunehmend verwischen.

\section{Befürchtete Multimorbidität soll sektorenübergreifend angegangen werden}

Segregation medizinischer Sektoren oder nicht, das ist auch ein Thema bei der Diskussion um eine erwartete medizinische Herausforderung der Zukunft: der Polybzw. Multimorbidität, die sich neuerdings einer speziellen Wahrnehmung erfreut. Aufgrund der gestiegenen Lebenserwartung wird allgemein mit einem starken Anstieg multimorbider Krankheitszustände in der Bevölkerung gerechnet. Jeder vierte über 70-Jährige leide an fünf oder mehr gleichzeitig behandelten Erkrankungen, so die Berliner Medizinsoziologin und 
Gerontologin Adelheid Kuhlmey auf der HelsanaTagung. Der Leiter der Klinik und Poliklinik für Innere Medizin am UniversitätsSpital Zürich Edouard Battegay illustrierte dies auf der SAMW-Tagung mit plastischen Beispielen, etwa der 79-jährigen Patientin mit Hypertonie, Diabetes, Arthrosen, Osteoporose und chronischer Bronchitis. Die mit der Multimorbidität verbundene Polypharmazie schaffe eine Vielzahl ineinander verzahnter Variablen, die vom einzelnen Arzt kaum mehr überblickt werden könne. Medikationen und Krankheiten interagierten. In die elektronische Krankengeschichte eines über mehrere Monate hospitalisierten polymorbiden Patienten hätten 71 Ärztinnen und Ärzte Einträge gemacht. 121 Personen aus 38 Organisationseinheiten der Klinik hätten in das Dossier Einsicht genommen. Als Lösung, die aus der Polymorbidität entstehenden Herausforderungen zu meistern, plädierte Battegay für ein Verständnis von Medizin als ärztliches Teamwork, fragte sich aber zweifelnd, ob «wir für den Teamsport richtig trainiert sind».

Vernetzung, Kooperation und Abstimmung statt Segregation wird geradezu als Zauberwort zur Lösung erwarteter Probleme und wichtigste Zukunftsaufgabe der Medizin verstanden, weit über die Ärzteschaft hinaus. Chronische Krankheiten, so Claudia Steurer-Stey vom Institut für Hausarztmedizin der Universität Zürich, bedürften einer koordinierten Betreuung, in der die beteiligten Leistungserbringer untereinander abgestimmt sind. Nicht nur Grundversorger und Spezialisten, sondern auch das Gemeinwesen sowie Pflege- und Betreuungsinstitutionen müssten einbezogen werden. Die Helsana-Hauptrednerin Kuhlmey sieht dies z.B. in Chronic-Care-Modellen mit multiprofessionellen Care-Teams umsetzbar, bei denen ambulante therapeutische und palliative Versorgung, Beratung, professionelle und private Pflege verzahnt seien. So würden Schnittstellen überwunden und sektorale Konkurrenz werde verhindert.

Unterschiedliche Ansichten bestanden allerdings zur Frage, wer im Zentrum solcher Betreuungsmodelle stehen solle. Enthusiasten der Hausarztmedizin sehen die Koordination bei den medizinischen Grundversorgern. Kuhlmey konnte sich auch den Berufsbereich der «Nurse Practitioners» vorstellen, solange sie sich mit den Hausärzten abstimmten. Die Aufgabe müsse auch nicht zwingend einer Berufsgruppe zugedacht werden.

Nicht zuletzt seien auch das Selbstmanagement der Patienten und ihre Krankheitskompetenz, ja das Entwickeln einer eigenen Expertenrolle unter den chronisch erkrankten Patienten von steigender Bedeutung. Wenn informierte Patienten ein Asthma bronchiale früh genug selbst erkennen, so ein Beispiel von Steurer-Stey, lassen sich Hospitalisationen verhindern. Dahinter stehe ein Ansatz, der die Patienten durch aktive und ausdauernde Integration zu Partnern im therapeutischen Prozess macht.

Zwei reichlich unterschiedliche Themen in der Diskussion um die Medizin der Zukunft legen damit ein Gemeinsames nahe: Die Medizin wird sich weniger im Ziehen von Grenzen finden als darin, diese Grenzen durchlässiger zu machen. In der Diskussion um die Polymorbidität werden das Überspringen von Berufsgrenzen und die Vernetzung der beteiligten Gruppen als wichtigste Lösungsansätze verstanden. Ein genauerer Blick auf das «Enhancement» zeigt, dass dieses, spätestens jenseits von Lifestyle-Resorts, verwoben in die gesamte Medizin und den aussermedizinischen Alltag ist. Ist ein drohender Arbeitsplatzverlust eine medizinische oder eine aussermedizinische Indikation für das Verschreiben eines Neuroenhancers zur Leistungssteigerung oder zur Bekämpfung von Depressionen? Wer will hier eine eindeutige Grenze ziehen? Enhancement erweist sich damit als ein Phänomen, an dem ablesbar ist, wie die Medizin mit dem Rest der Gesellschaft vernetzt ist und auf das «die Medizin» nicht alleine als vermeintlich abgeschlossenes System reagieren kann. Eine «reine», abgrenzbare und in separierte Felder und Aufgaben aufgeteilte Medizin ist eher Wunsch als Wirklichkeit und wohl noch weniger eine Lösung.
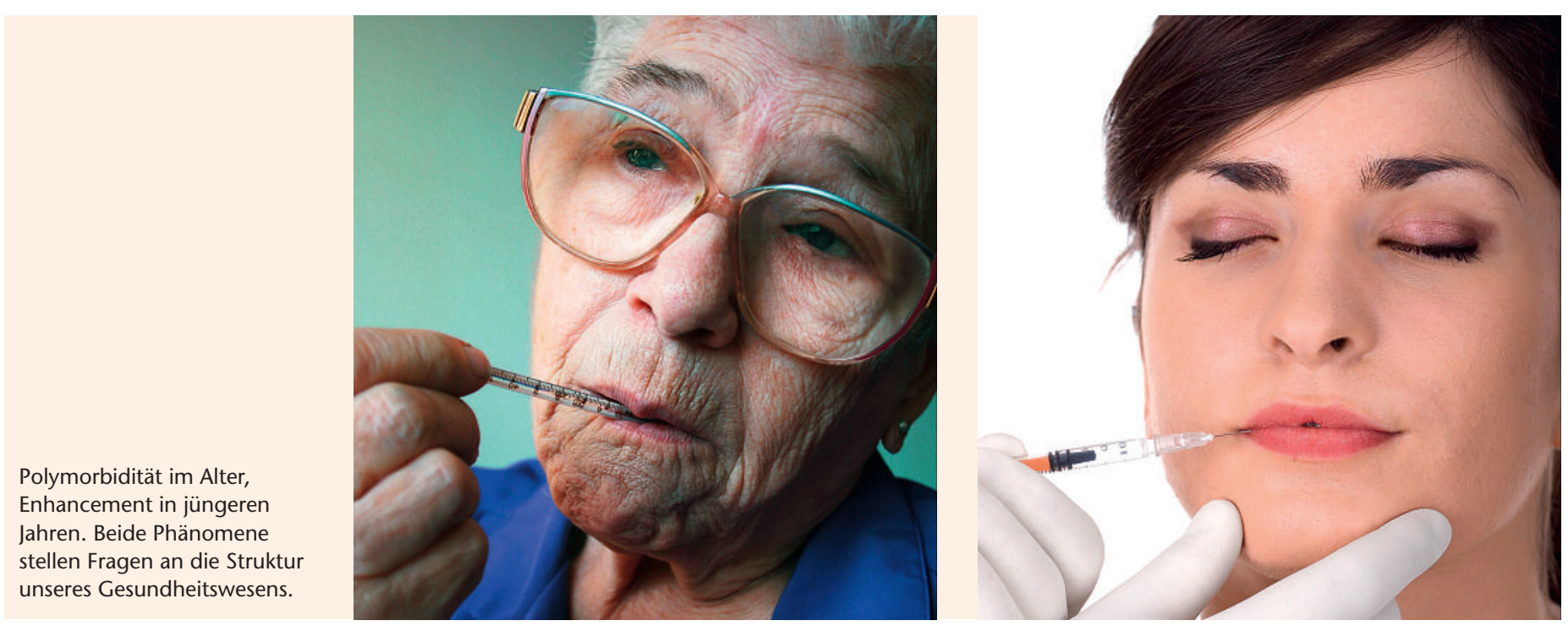

Polymorbidität im Alter, Enhancement in jüngeren Jahren. Beide Phänomene unseres Gesundheitswesens. stellen Fragen an die Struktur 